\title{
The Significance of Heat Shock Protein 70 Expression in Benign Thyroid Nodules During Thermal Ablation
}

\author{
Lei Yan, MM ${ }^{a, b, 1}$, Jianquan Zhang, MD ${ }^{a, I, *}$, Jianguo Sheng, MM ${ }^{a}$, Hang Zhang, MM ${ }^{a}$, Zongping Diao, MM ${ }^{a}$, \\ Jianming Zheng, PhD ${ }^{c}$ \\ ${ }^{a}$ Department of Ultrasound in Medicine, Changzheng Hospital, Naval Medical University (Second Military Medical University), \\ Shanghai, China; ${ }^{b}$ Department of Ultrasound in Medicine, No. 904 Hospital of PLA, Suzhou, Jiangsu, China; ${ }^{c}$ Department of Pathology, \\ Changhai Hospital, Naval Medical University (Second Military Medical University), Shanghai, China \\ Received March 29, revision received May 16; accepted June 1. \\ ${ }^{1}$ Lei Yan and Jianquan Zhang contributed equally to this study.
}

Objective: To investigate the change of HSP70 expression after microwave ablation of benign thyroid nodules.

Method: Ultrasound-guided microwave ablation (MWA) was conducted for treating 60 benign solid thyroid nodules in 60 patients. Three different region of interest (ROI), named as central zone, marginal zone and transitional zone, were artificially set in each nodule for quantitative investigation of HSP70 expression by using the immunohistochemistry (IHC) method based on the US-guided core-needle biopsy (CNB) technique. As a longitudinal comparative study design, each ROI experienced twice histological sampling twice by using CNB before and after MWA. IHC semi-quantitative determination of HSP70 expression was compared among the specimen from the three ROIs in a same nodule before ablation and between the specimen from the same ROI before and after ablation respectively as well.

Results: Of the 60 solid thyroid nodules, either in the central zone or in the marginal zone, HSP70 expression did not experienced significant change $(P>0.05)$ from before ablation to after ablation, however, a significant upgrading of HSP70 expression developed in the transitional zone after ablation $(P<0.05)$. After ablation, the transitional zone had a higher level of HSP70 than that in both the central and marginal zones $(P<0.05)$ in a same nodule, while the level of HSP70 was not found significantly different between the central zone and marginal zone $(P>0.05)$.

Conclusion: Thermal coagulation by MWA stimulates the upgrading of HSP70 expression in the heated tissue of benign thyroid nodules, but in a way of regional discrepancies. The remarkable increased expression of HSP70 in the transitional zone tissue might originate from a slower and less intensive temperature rise along with the attenuation of microwave radiation, and it is postulated to protect normal glandular tissue against heat damage. Fast and intensive temperature rise within the nodule causes more intensive and massive thermal coagulation changes and weakens the adequate expression and proper protective function of HSP70 in those two areas.

Key Words: Microwave ablation; Heat shock protein 70; Thyroid nodule; Immunohistochemistry

Advanced Ultrasound in Diagnosis and Therapy 2019;03:103-108

DOI: 10.37015/AUDT.2019.190817

Percutaneous thermal ablation therapy (TAT) for the treatment of thyroid nodules has aroused increasing attention [1] due to accurate location, minimal trauma, complete inactivation, protection of the thyroid function

* Corresponding Author: Department of Ultrasound in Medicine, Changzheng Hospital, 415 Fengyang Road, Shanghai, China.
e-mail: wintersnow9090@sina.com

2576-2508/O AUDT 2019 • http://www.AUDT.org

This is an open-access article distributed under the terms of the Creative Commons Attribution 4.0 International license, which permits unrestricted use, distribution and reproduction in any medium provided that the original work is properly attributed. 
and avoidance of visible cervical scars. The greatest concern in the wider spread of TAT is whether thermal ablation would cause injury to the normal thyroid tissue and the adjacent cervical structures. To evade this problem, various artificial means such as the "hydrodissection technique" method have been advocated as assisted measures. However, whether the body itself also plays some protective role in this process, and if so how it exerts this protective effect remain to be explored. Heat shock protein (HSP) is a protein produced by the organism spontaneously upon thermal stimulation. Studies have demonstrated that HSP can actively protect against stimulation and repair stimulation-induced injuries. Among various HSP subtypes, HSP70 has aroused our interest. It was reported that the expression of HSP70 was enhanced in thyroid tumors [2]. However, there is no study reporting whether HSP70 can also play a self-protective role in the process of thermal ablation for the treatment of thyroid nodules. The aim of the present study was to explore HSP70 expression changes in thyroid nodules and adjacent structures before and after TAT, analyze rules of such changes, and discuss the potential significance.

\section{Patients and Methods}

\section{Patients}

Sixty patients with a single solid thyroid nodule were selected from patients with thyroid nodules who received ultrasound-guided microwave ablation (MWA) therapy between September 2013 and February 2014 in Shanghai Changzheng Hospital (Shanghai, China). Preoperative puncture biopsy confirmed that all the 60 nodules were benign, including follicular adenoma in 32 cases and nodular goiter in 28 cases. The 20 male and 40 female patients ranged in age from 27 to 57 years with a median of 40 years. The research protocol was approved by the ethics committee of the hospital, and informed consent was obtained from all patients concerned.

\section{Instruments and Materials}

The instruments used in this study included Siemens Sequoia 512 with the transducer frequency of 7-14MHz; Microwave unit (KY-2000; Kangyou Medical, Nanjing, China) equipped with KY-2450A type microwave needle (external diameter $1.6 \mathrm{~mm}$ and the tip working length $10 \mathrm{~cm}$ ); and 16G-HS histological biopsy needle (Presia, Italy).

\section{Inclusion criteria of nodule for ablation}

The inclusion criteria were 1) completely solid nodules with the maximal diameter of $15-20 \mathrm{~mm}$ for the sake of covering the whole nodule at a single ablation and for the convenience of successful biopsy sampling; and 2) no diffuse thyroid disease out of the nodule to avoid abnormal basic HSP70 expression that may possibly arise from the underlying disease.

\section{Definition of the area of interest}

Knowing that the temperature during TAT tends to decrease progressively from the center of the ablation needle passage to the periphery of nodule in a gradientdependent manner [3], we postulated that different thermal doses may cause the expression of HSP70 to undergo gradient changes from the center to the periphery. In addition, a certain dimension of normal thyroid tissue beyond the nodule may also be affected by thermal stimulation, thus promoting HSP70 expression and affecting the surrounding normal tissue. To investigate HSP70 expression in different intra-nodular areas and extra-nodular adjacent tissues, three areas of interest were artificially set for each nodule: 1) the central zone, which refers to the ablation needle passage and adjacently aside tissue; 2) the marginal zone, which refers to $3 \mathrm{~mm}$-dimension area inside the ultrasonic margin of the nodule; and 3) the transitional zone, which refers to $5 \mathrm{~mm}$-dimension area of normal thyroid tissue outside the ultrasonic margin of the nodule.

\section{Ablation methodology}

Preoperative ultrasonography was performed to learn about the location, boundary, configuration and internal echogenic features distribution of the nodule. Color Doppler ultrasound and contrast-enhanced ultrasound (CEUS) were performed to learn about blood supply to the nodule. With the neck hyper-extended, the cervical skin of the patient was draped and sterilized routinely, and the puncture site was anesthetized locally with $5 \mathrm{ml} 2 \%$ lidocaine $+15 \mathrm{ml}$ normal saline as the hydrodissection fluid to protect the trachea, common carotid artery and recurrent laryngeal nerve. Subsequently, the KY-2450A microwave needle electrode was used to perform multi-point and multi-plane microwave ablation using a $35-45 \mathrm{w}$ power. Postoperative contrast-enhanced ultrasound showed that filling in the ablation area disappeared completely.

\section{Pathological sampling and staining}

Using 16G-HS histological biopsy needle, tissues were sampled from the central, marginal and transitional zones of the nodule respectively before and immediately after TAT, fixed in $4 \%$ formaldehyde, and stained by immunohistochemistry using the Envision two-step method. Rabbit anti human HSP70 polyclonal antibody (specific to all HSP70 members) was used as the reagent and diluted at 1:100. 


\section{Interpretation of the pathological images}

Three different fields were selected randomly from each immunohistochemical slide $(\times 200)$ to read the positive intensity and positive rate of HSP70 expression by a senior and experienced pathologist. The HSP staining result (the sum of the score of staining intensity and the percentage of positive cells) was calculated by the semi-quantitative method. By subtracting the background staining, the staining degree of most cells constitutes the concept of staining intensity and was scored as follows: no staining, 0 ; light or mild yellow, 1; dark yellow or brown yellow, 2; and brown or black brown, 3. The concept of percentage of staining positive cells refers to the number of stained cells per 100 cells in each of the 5 different visual fields of each immunohistochemical slide $(\times 400)$, and the mean percentage value upon the sum of five visual fields was scored as follows: $0-5 \%$, $0 ; 6-25 \%, 1 ; 26-50 \%, 2 ; 51-75 \%, 3$; and $>75 \%, 4$. The total scores of HSP70 expression are the sum of staining intensity score and percentage of staining positive cells score and was ranked as follows: no expression $(-),<$ 2; weak expression $( \pm), 2 \sim 3$; moderate expression $(+)$, 4 5; and high expression (++), 6 7. No expression and weak expression were merged as the negative group, and moderate expression and high expression were merged as the positive group.

\section{Statistical analysis}

Data analysis was carried out using SPSS20.0 software. Comparison of the positive HSP expression rate and intensity between two groups was performed by chi-square test of four-fold table. Significance level was defined as $P<0.05$.

\section{Results}

\section{HSP70 expression}

Immunohistochemical staining showed that positive HSP70 expression was mainly located in the cytoplasm of thyroid follicular epithelial cells, and only a small proportion was located in the nucleus, both presenting a yellow to brown color. Of the 60 benign thyroid nodules, different degrees of HSP70 expression were detected before and after TAT. In most post-TAT specimens, high HSP70 expression was observed in the transitional zone of the nodule, and moderate HSP70 expression was detected in the central and marginal zones (Fig. 1). In addition, positive staining was present in colloid within follicular cavities of some specimens. Knowing that colloid in follicular cavities contains HSP70, it was excluded from calculation of positive cells.
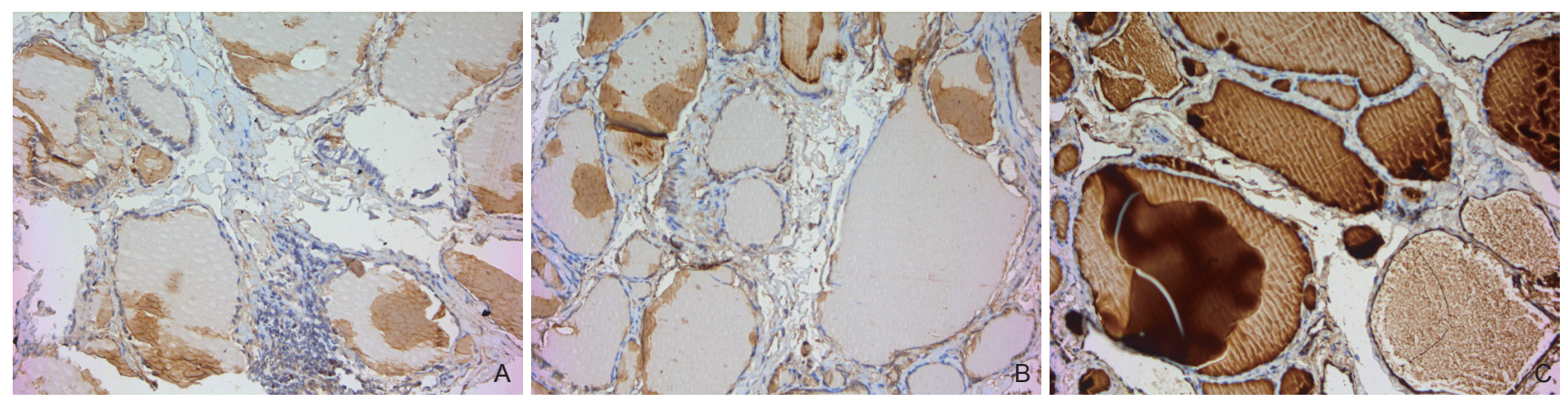

Figure 1 Expressions of HSP70 in different zones of thyroid adenoma shortly after thermal ablation $(\times 200)$. (A) Weak expressions of HSP70 in the central zone; (B) weak expressions of HSP70 in the marginal zone; (C) intensive expressions of HSP70 in the transitional zone.

\section{Correlation between TAT and HSP70 expression}

Comparison of the positive expression rate and intensity of HSP70 in various zones of the thyroid nodule before and after TAT showed that there was no significant difference in the central and marginal zones respectively before and after TAT (both $P>0.05$ ) (Fig. 2-3), while the transitional zone underwent stronger positive expression of HSP70 after TAT than before TAT (Table 1)(Fig. 4).

\section{Regional distribution of HSP70 expression after TAT}

Paired comparison of post-TAT HSP70 expression in the central, marginal and transitional zones showed that the increment of HSP70 expression in the transitional zone was significantly stronger than that either in the central zone or marginal zone $(P=0.000)$, but the increment was not significantly different between the central zone and marginal zone $(P=0.853)$.

\section{Discussion}

Ultrasound-guided TAT has been used in the treatment of both benign and malignant tumors in the liver, pancreas, lung, thyroid gland, parathyroid gland, mammary gland, salivary gland and lymph nodes [411]. TAT works by inserting the needle electrode into the tumor to quickly cauterize and necrotize the tumor tissue 


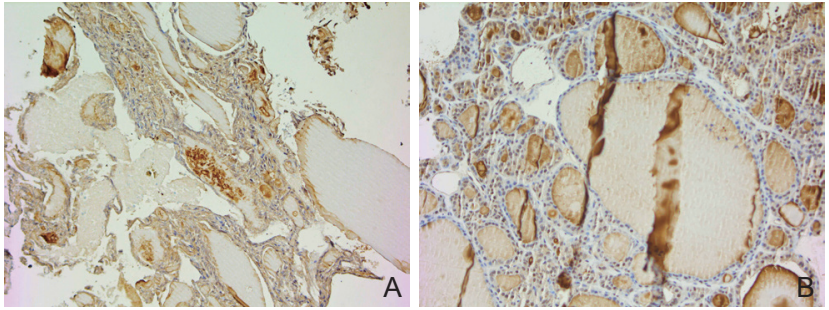

Figure 2 Expressions of HSP70 in central zones of thyroid adenoma before and shortly after thermal ablation $(\times 200)$. (A) weak expressions of HSP70 in the central zone before ablation; (B) weak expressions of HSP70 in the central zone shortly after ablation.

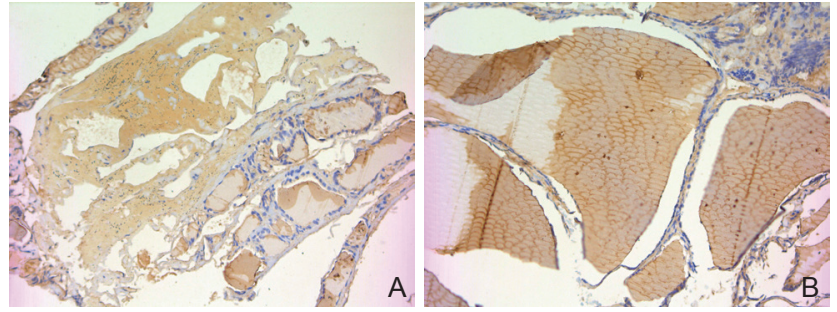

Figure 3 Expressions of HSP70 in central zones of nodular goiter before and shortly after thermal ablation $(\times 200)$. (A) week expressions of HSP70 in the marginal zone before ablation; (B) week expressions of HSP70 in the marginal zone shortly after ablation.

Table 1 Expressions of HSP70 in the same zones of benign thyroid nodules before and after thermal ablation

\begin{tabular}{|c|c|c|c|c|c|c|c|c|c|c|c|c|c|}
\hline \multirow{3}{*}{ Group } & \multirow{3}{*}{$n$} & \multicolumn{5}{|c|}{ Before ablation } & \multicolumn{5}{|c|}{ After ablation } & \multirow{3}{*}{$\chi^{2}$ value } & \multirow{3}{*}{$P$ value } \\
\hline & & \multicolumn{4}{|c|}{ expression intensity } & \multirow{2}{*}{$\begin{array}{l}\text { positive } \\
\text { rate }\end{array}$} & \multicolumn{4}{|c|}{ expression intensity } & \multirow{2}{*}{$\begin{array}{l}\text { positive } \\
\text { rate }\end{array}$} & & \\
\hline & & - & \pm & + & ++ & & - & \pm & + & ++ & & & \\
\hline $\mathrm{CR}$ & 60 & 7 & 22 & 26 & 5 & $51.67 \%$ & 0 & 24 & 30 & 6 & $60.00 \%$ & 0.839 & 0.358 \\
\hline $\mathrm{BR}$ & 60 & 15 & 15 & 24 & 6 & $50.00 \%$ & 0 & 25 & 25 & 10 & $58.33 \%$ & 0.839 & 0.360 \\
\hline TR & 60 & 18 & 26 & 13 & 3 & $43.33 \%$ & 0 & 7 & 18 & 35 & $88.33 \%$ & 35.512 & 0.000 \\
\hline
\end{tabular}

CR: central zone; BR: marginal zone; TR: transitional zone

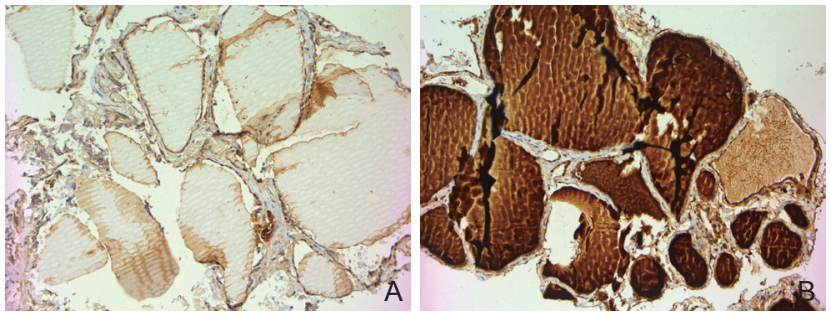

Figure 4 Expressions of HSP70 in transitional zones of thyroid adenoma before and shortly after thermal ablation $(\times 200)$. (A) weak expressions of HSP70 in the transitional zone before ablation; (B) strong expressions of HSP70 in the transitional zone shortly after ablation.

by the radiofrequency or microwave generated from the generator. The advantages of TAT lie in accurate location, multi-point treatment of the lesion, minimal invasion and the absence of visible skin scars. The efficacy of TAT on thyroid nodules should be assessed from two aspects: the therapeutic effect on the focal lesions and the potential adverse affects on adjacent normal structures. Previous studies have mainly focused on inactivation of the nodule by ATA, and studies concerning the influence of ATA on adjacent normal tissues have been limited to liver tumors, saying that HSP expression was increased markedly in the surrounding tissue of the liver tumor after TAT [5]. Thyroid gland is an indispensible endocrinal organ for human beings. As it is in close proximity with the blood vessels, trachea, nerves, parathyroid gland and other important structures, failure to protect the normal thyroid tissue and surrounding structures may result in voice alteration, brachia plexus injury, tumor rupture, permanent thyroid dysfunction, hematoma, skin burn, vomiting and other complications [12]. The primary purpose of thyroid nodule TAT is to inactivate the lesion quickly and accurately without causing injury to the surrounding normal structures. Some studies in China [8] pioneered the use of "hydro-dissection technique" to protect the structures surrounding thyroid gland. However, this technique lacks the ability to protect the normal thyroid tissue surrounding the thyroid nodule for ablation. There is also no study reporting a method that can protect the normal tissue within the thyroid gland. In the present study, we used HSP70 as an observation index to assess the possible influence of TAT on the thyroid gland, knowing that HSP70 is a protein commonly used to evaluate the protective effect against thermal injury and can be readily detected in most laboratories.

The HSP family is a unique group of stress proteins extensively existing in prokaryotic and eukaryote cells. They are produced upon heat, hypoxia and chemical stimulations [13]. Being a molecular chaperon, HSP woks on protein transport and assembly, and correct folding of peptide chains, thus playing an important role in protecting cells, and ultimately affecting cells survival [14]. Studies in recent years have demonstrated that HSP is associated with cell apoptosis [15]. HSP70 is the most important member of the HSP family that has been widely investigated. Apart from the common functions of the other members of the HSP family, HSP70 also has various other biological functions, including involvement in regulating immunity and attenuating inflammatory responses. In addition, HSP70 is also associated with 
viral infection and specific/non-specific anti-tumor immunity [16-18]. The amount of HSP expression is relatively small under normal physiological conditions. However, various physiochemical stimulations can trigger the expression of HSP70 to protect cells by quickly increasing its content within cells. In addition, tumors themselves are a type of stimulation than can increase the expression of HSP70. The expression level of HSP is different in benign and malignant tumors [19]. HSP exists in both thyroid follicular epithelial cells and follicular cavities. HSP70 in follicular cavities is also synthesized in follicular epithelial cells, and releases extracellularly by means of exocytosis.

During the process of TAT, heat is transmitted from the central needle passage to the periphery zones in a gradually decreasing manner, and therefore the temperature keeps the highest within the needle tract, which is also the place that first receives the heat stimulation. Theoretically, the increase in HSP expression should be earliest triggered in follicular epithelial cells in this area, and with the lapse of time it is gradually provocated in the marginal and transitional zones. Thus in theory, the central zone should be the place showing the highest HSP expression. However, the present study found that there was no significant increase in post-TAT HSP expression in the central and marginal zones as compared with that before TAT. We postulate that this may be because the quick generation and strong intensity of microwave heat cause such fast coagulation of tissue in the central zone and marginal zone that they have no adequate time and ability to synthesize HSP before being coagulated. Transitional zone refers to the normal thyroid gland tissue within $5 \mathrm{~mm}$ dimension outside the ultrasonic boundary of the nodule, and the follicular tissue there is probably beyond effective thermal coagulation, and thus it remains viable and has adequate time and viability to synthesize HSP70. Considering its natural function of self-protection, we think that the significant increment of HSP70 expression in the transitional zone may play a central role for the normal extra-nodular thyroid tissue against thermal damage. It helps to make a point that thermal therapy by using MWA is a safe modality for treatment of benign thyroid nodules.

Studies on spatial distribution of HSP expression in benign thyroid nodules after TAT showed that the microwave temperature decreased gradually from the center to the periphery [3], and along with the gradual decrease of temperature, the degree of histological denaturation decreased gradually. We postulated that HSP expression within the thyroid tissue should also have the gradual-decrease feature. However, the present study showed that HSP expression in different zones did not show a tendency of gradual and gradient decrease.

\section{Conclusion}

The present study revealed that thermal coagulation by MWA stimulates the upgrading of HSP70 expression in the heated tissue of benign thyroid nodules, but with regional discrepancies. Although the study did not reach a clear understanding of how the upgrading of HSP70 expression influences the thermal efficacy of the target lesions, we found the marked increased expression of HSP70 in the transitional zone tissue might originate from a slower and less intensive temperature rise along with the attenuation of microwave radiation, which is postulated to protect normal glandular tissue against heat damage. Fast and intensive temperature rise within the nodule causes more intensive and massive thermal coagulation changes and weakens the adequate expression and proper protective function of HSP70 in those two areas.

\section{Funding}

This work was supported by National Natural Science Foundation of China (Grant No. 81171436)

\section{Conflict of Interest}

The authors have no conflict of interest to declare.

\section{References}

[1] Zhang JQ, Yan L, Diao ZP, Chen HQ, Cheng J. Potential threat of tracheal diverticulum to thermal ablation treatment of thyroid nodule. AUDT 2019;01:006-011.

[2] Soudry E, Stern Shavit S, Hardy B, Morgenstern S, Hadar T, Feinmesser R. Heat shock proteins HSP90, HSP70 and GRP78 expression in medullary thyroid carcinoma. Ann Diagn Pathol 2017;26:52-56

[3] Zhang JQ, Diao ZP, Lu F, Sheng JG. Temperature gradient and elasticity gradient over the microwave-heated region in porcine liver in vitro. Chin J Ultrasonogr 2012;21:799-802. [In Chinese]

[4] Li M, Li Z, Gao Y, Tian J, Chen M, Dong J. Safety and efficacy of percutaneous radiofrequency ablation combined with percutaneous ethanol injection for hepatocellular carcinoma in high-risk locations. AUDT 2018;2:05-16.

[5] Ahmed M, Kumar G, Gourevitch S, Levchenko T, Galun E, Torchilin $\mathrm{V}$, et al. Radiofrequency ablation (RFA)-induced systemic tumor growth can be reduced by suppression of resultant heat shock proteins. Int J Hyperthermia 2018;34:934-942.

[6] Rustagi T, Chhoda A. Endoscopic radiofrequency ablation of the pancreas. Dig Dis Sci 2017;62:843-50.

[7] Ito T, Oura S, Nagamine S, Takahashi M, Yamamoto N, Yamamichi N et al. Radiofrequency ablation of breast cancer: a retrospective study. Clin Breast Cancer 2018;18:e495-e500.

[8] Zhang JQ, Ma Na, Xu B, Liu C, Song JL, Diao ZP. Methodology of percutaneous bi-polar radiofrequecy ablation of thyroid adenomas under ultrasound guidance and monitoring. Chin J Ultrasonogr 2010;19:861-865. [In Chinese]

[9] Liu C, Zhang JQ, Xiao L. Clinical application of ultrasound-guided percutaneous radiofrequency ablation for parathyroid adenoma. Acad 
J Sec Mil Med Univ 2013;34:371-4. [In Chinese]

[10] Zhang JQ, Lei Y, Chen HQ, Wu ZZ. Cyto-inactivation instantly induced by microwave ablation on thyroid nodules. Natl Med J China 2018;98:3524-7. [In Chinese]

[11] Zhang JQ, Qin ZF, Jiang JJ, Meng J. Percutaneous radiofrequency ablation combined with microwave coagulation therapy under contrast-enhanced ultrasound guidance for treatment of melanoma metastasis to lymph nodes:a case report . Acad J Sec Mil Med Univ,2006;27:1364-7. [In Chinese]

[12] Baek JH, Lee JH, Sung JY, Baek J, Kim K T, Sim J, et al. Complications encountered in the treatment of benign thyroid nodules with US-guided radiofrequency ablation: amulticenter study. Radiology 2012;262:335-42.

[13] Wu J, Liu T, Rios Z, Mei Q, Lin X, Cao S. Heat Shock Proteins and Cancer. Trends Pharmacol Sci 2017;38:226-56.

[14] Chen B, Feder ME, Kang L. Evolution of heat-shock protein expression underlying adaptive responses to environmental stress. Mol Ecol 2018;27:3040-54.
[15] Zhang XH, Wu H, Tang S, Li QN, Xu J, Zhang M, et al. Apoptosis in response to heat stress is positively associated with heatshock protein 90 expression in chicken myocardial cells. J Vet Sci 2017;18:129-40.

[16] Chen T, CaoX. Stress formaintaining memory: HSP70 as amobile messenger for innate and adaptive immunity. Eur J Immunol, 2010; 40: 1541-44.

[17] Dodd K, Nance S, Quezada M, Janke L, Morrison JB, Williams RT, et al. Tumor-derived inducible heat-shock protein 70 (HSP70) is an essential component of anti-tumor immunity. Oncogene 2015;34:1312-22.

[18] Wang XP, Wang QX, Lin HP, Xu B, Zhao Q, Chen K. Recombinant heat shock protein 70 functional peptide and alpha-fetoprotein epitope peptide vaccine elicits specific anti-tumor immunity. Oncotarget 2016;7:71274-84.

[19] Calderwood SK. Heat shock proteins and cancer: intracellular chaperones or extracellular signalling ligands? Philos Trans $R$ Soc Lond B Biol Sci 2018;373(1738): 20160524. 